\title{
HS-104, a PI3K inhibitor, enhances the anticancer efficacy of gemcitabine in pancreatic cancer
}

\author{
KYUNG HEE JUNG ${ }^{1,2^{*}}$, HONG HUA YAN ${ }^{1 *}$, ZHENGHUAN FANG ${ }^{1}$, MI KWON SON ${ }^{1}$, \\ HYUNSEUNG LEE ${ }^{1}$, SUNGWOO HONG ${ }^{3}$ and SOON-SUN HONG ${ }^{1}$ \\ ${ }^{1}$ College of Medicine, Inha University, Incheon 400-712; ${ }^{2}$ College of Pharmacy, Chonnam National University, \\ Gwang-ju 300; ${ }^{3}$ Department of Chemistry, Korea Advanced Institute of Science and \\ Technology (KAIST), Daejeon 305-701, Republic of Korea
}

Received January 29, 2014; Accepted April 3, 2014

DOI: $10.3892 /$ ijo.2014.2435

\begin{abstract}
Gemcitabine has limited clinical benefits for pancreatic cancer patients. The phosphatidylinositol-3-kinase (PI3K)/ Akt signaling pathway is important in cell proliferation and survival, and is frequently dysregulated in pancreatic cancer. To obtain insights into novel therapeutic strategies for treating pancreatic cancer, we investigated whether HS-104, a novel PI3K inhibitor, in combination with gemcitabine would show a synergistic effect in pancreatic cancer. We first evaluated the effect of gemcitabine alone or in combination with HS-104 on cell viability. When administered together, the two drugs synergistically inhibited the viability of AsPC-1 and PANC-1 cells, and decreased mitochondrial membrane potential, thereby inducing apoptosis. Compared to the treatment with either drug alone, the combination treatment resulted in apoptosis accompanied by increased levels of cleaved caspase-3 and Bax. These results were consistent with decreased expression of p-Akt, p-mTOR, p-Mek and p-Erk. Moreover, the combination treatment inhibited blood vessel formation in a Matrigel plug assay in mice. Furthermore, in vivo, the combination significantly inhibited tumor growth and enhanced apoptosis by increasing the number of TUNEL-positive cells, and cleaved caspase- 3 together with decreasing the expression of angiogenesis- and proliferation-related effectors such as CD34 and PCNA in tumor tissues, compared with each drug alone. Taken together, our study demonstrates that the combination of gemcitabine and HS-104 had a synergistic anticancer effect and inhibited the PI3K/Akt and RAF/Mek pathways on pancreatic cancers. On the basis of our results, we suggest that
\end{abstract}

Correspondence to: Professor Soon-Sun Hong, Department of Biomedical Sciences, College of Medicine, Inha University, 3-ga, Sinheung-dong, Jung-gu, Incheon 400-712, Republic of Korea E-mail: hongs@inha.ac.kr

*Contributed equally

Key words: gemcitabine, phosphatidylinositol-3-kinase, pancreatic cancer the combination of these two drugs may be considered as a new therapeutic regimen for treating pancreatic cancer.

\section{Introduction}

Pancreatic cancer is the fourth leading cause of cancer-related death, with a 5-year survival rate below 5\% (1). The main reasons for this poor prognosis include invasive behavior and multifactorial chemoresistance of the cancer. Gemcitabine is considered as the standard first-line chemotherapeutic agent for the treatment of advanced pancreatic cancer and has offered some relief to affected patients over the past two decades (2). However, its efficacy remains moderate and the median overall survival times range from 5 to 8 months. Furthermore, pancreatic cancer has been reported to easily acquire resistance to gemcitabine after a few cycles of administration. For this reason, numerous studies have sought to increase the efficacy of chemotherapy by combining gemcitabine with other agents. However, administration of other chemotherapeutics, monoclonal antibodies, or radiation in addition to gemcitabine has not resulted in any meaningful improvement in the survival of pancreatic cancer patients (3-5). Thus, pancreatic cancer remains a refractory cancer and one of the most challenging problems in oncology (6). These disappointing results necessitate novel combination therapies to improve the survival of pancreatic cancer patients and to identify novel molecular targets in this advanced disease (6).

The phosphatidylinositol-3-kinase (PI3K)/Akt signaling pathway plays an important role in biological processes including cell proliferation, differentiation and survival $(7,8)$. The PI3K pathway is activated by various extracellular signals and leads to the phosphorylation of Akt and its downstream effectors (9). When Akt is phosphorylated, it in turn, phosphorylates proteins such as mTOR and 4EBP1 (9). Dysregulation of PI3K/Akt pathway is a prominent feature of pancreatic cancers, because of the high prevalence of abnormalities that regulate this pathway, including K-ras mutations that occur in approximately $90 \%$ of cases, and increased expression of EGFR, which is an important regulator in PI3K/Akt signaling (10-13). Previous studies have also shown that the activation of the PI3K/Akt signaling pathway is a result of aberrant expression of PTEN in pancreatic cancer cell lines $(14,15)$. In 
addition, Akt is reported to be constitutively overexpressed in various pancreatic cancer cell lines (16). Importantly, the $\mathrm{PI} 3 \mathrm{~K} / \mathrm{Akt}$ pathway has been reported as a crucial factor in the conferring of chemoresistance to gemcitabine in pancreatic cancer (17-20). Furthermore, inhibition of the PI3K/Akt pathway by LY294002 or wortmannin enhances gemcitabineinduced apoptosis in human pancreatic cancer cells (17). Considering these findings, it is likely that the activation of the PI3K/Akt pathway has an important role in the aggressive clinical features of pancreatic cancer. Although PI3K/Akt inhibitors have therapeutic potential when used either alone or in combination with gemcitabine in the treatment of pancreatic cancer, severe cytotoxicity has been observed in preclinical animal studies, leading to their limited use in clinical trials. Thus, the PI3K/Akt pathway is still a promising target for therapeutic intervention in human pancreatic cancer, and the identification of effective novel PI3K inhibitors to enhance clinical efficacy is important.

HS-104 is a synthetic PI3K inhibitor belonging to the imidazopyridine derivative class (21). In our previous study, we reported that HS-104 suppressed tumor proliferation and angiogenesis in various cancers $(22,23)$. On the basis of our previous findings, we decided to combine gemcitabine and HS-104, which may represent a major advantage over traditional chemotherapies. The effect of these combinations on cancer cell growth, death and angiogenesis is unknown. In this study, we tested the hypothesis that co-treatment of the new PI3K inhibitor HS-104 could enhance the anticancer effect of gemcitabine by blocking multiple pathways, including the PI3K/Akt signaling pathway, thereby halting the progression of pancreatic cancer.

Gemcitabine and HS-104 acted synergistically to induce apoptosis and inhibit cell growth or angiogenesis in pancreatic cancer. This combination treatment led to a stronger inhibitory effect on cell viability than did treatment with either drug alone. Our findings suggest that a combination treatment comprising gemcitabine and HS-104 may be a promising therapeutic strategy for pancreatic cancer.

\section{Materials and methods}

Cells and materials. Human pancreatic cancer cell lines (AsPC-1 and PANC-1) used in this study were purchased from the American Type Culture Collection (ATCC, Manassas, VA). AsPC-1 cells were grown in Roswell Park Memorial Institute-1640 (RPMI-1640) medium and PANC-1 cells were grown in Dulbecco's modified Eagle's medium (DMEM). Both types of media were supplemented with $10 \%$ fetal bovine serum (FBS) and $1 \%$ penicillin/streptomycin. Cell cultures were maintained at $37^{\circ} \mathrm{C}$ in a $\mathrm{CO}_{2}$ incubator with a controlled humidified atmosphere composed of $95 \%$ air and $5 \% \mathrm{CO}_{2}$. Gemcitabine [4-amino-1-(2-deoxy-2,2-difluoro- $\beta$-D-erythro-pentofuranosyl) pyrimidin-2(1H)-on] was purchased from LTK Corporation (Wilmington, DE), and HS-104 [N-(5-(3-(3-methyl-1,2,4oxadiazol-3-yl)imidazo[1,2-a]pyridin-6-yl)pyridin-3-yl) benzenesulfonamide], a new PI3K inhibitor, was synthesized according to our previous methods (21).

Cell growth assay. The growth rate of gemcitabine- or HS-104-treated cells was determined using a 3-(4,5-dimethylthiazol-2-yl)-2,5-diphenyl tetrazolium bromide (MTT) assay.
Exponentially dividing cells (PANC-1, 6,000 cells; AsPC-1, 8,000 cells) were plated in 96-well plates. The following day, the medium was removed and the cells were treated with either dimethyl sulfoxide (DMSO) and/or DDW as a control or gemcitabine $(1 \mu \mathrm{M})$ and/or HS-104 $(1,5$ and $10 \mu \mathrm{M})$. After the cells were incubated for $72 \mathrm{~h}, 20 \mu 1$ of MTT solution $(2 \mathrm{mg} / \mathrm{ml})$ was added to each well and the plate was incubated for another $4 \mathrm{~h}$ at $37^{\circ} \mathrm{C}$. The formazan crystals formed were dissolved in DMSO (100 $\mu \mathrm{l} / \mathrm{well})$ with constant shaking for $30 \mathrm{~min}$. The plate was then read using a microplate reader at a wavelength of $540 \mathrm{~nm}$. The median inhibitory concentration for cell growth $\left(\mathrm{IC}_{50}\right.$, the drug concentration at which cell growth was inhibited by $50 \%$ ) was assessed from the dose-response curves. The combination index $(\mathrm{CI})$, used to measure drug interaction between gemcitabine and HS-104, was calculated according to the Chou-Talalay method (24). Data were analyzed using the CalcuSyn software (Biosoft, Ferguson, MO). The CI index has been used for data analysis of two-drug combinations. Index values of $\mathrm{CI}<1, \mathrm{CI}=1$ and $\mathrm{CI}>1$ indicate synergism, additive effect and antagonism, respectively.

Western blot analysis. Total cellular protein content was extracted using a lysis buffer containing $1 \%$ Triton X-100, $1 \%$ Nonidet P-40, and the following protease and phosphatase inhibitors: aprotinin $(10 \mathrm{mg} / \mathrm{ml})$, leupeptin $(10 \mathrm{mg} / \mathrm{ml}$; Icn Biomedicals, Asse-Relegem, Belgium), phenylmethylsulfonyl fluoride (1.72 mM), $\mathrm{NaF}(100 \mathrm{mM}), \mathrm{NaVO}_{3}(500 \mathrm{mM})$, and $\mathrm{Na}_{4} \mathrm{P}_{2} \mathrm{O}_{7}(500 \mathrm{mg} / \mathrm{ml}$; Sigma-Aldrich, St. Louis, MO). The proteins were separated by sodium dodecyl sulfate-polyacrylamide gel electrophoresis (SDS-PAGE) and transferred onto nitrocellulose membranes. The blots were immunostained with the appropriate primary antibodies followed by secondary antibodies conjugated to horseradish peroxidase. Antibody binding was detected with an enhanced chemiluminescence reagent (Amersham Biosciences, Cambridge, MA). Antibodies against p-Mek $\left(\mathrm{Thr}^{286}\right)$, p-Erk $\left(\mathrm{Thr}^{202} / \mathrm{Tyr}^{204}\right)$, Erk, p-mTOR (Ser $\left.{ }^{2448}\right)$, mTOR, p-Akt $\left(\operatorname{Ser}^{473}\right)$, Akt, p-4EBP1, 4EBP1, PARP, cleaved caspase-3, Bcl-2, Bax and $\beta$-actin were purchased from Cell Signaling Technology (Danvers, MA).

Immunofluorescence. AsPC-1 cells were plated on 18-mm glass cover slips with RPMI-1640 medium and grown to approximately $70 \%$ confluence for $24 \mathrm{~h}$. The cells were treated with gemcitabine $(1 \mu \mathrm{M})$ and/or HS-104 $(5 \mu \mathrm{M})$ for $6 \mathrm{~h}$, washed twice with PBS, and fixed in an acetic acid: 70\% ethanol (1:2) solution for $10 \mathrm{~min}$ at $-20^{\circ} \mathrm{C}$. Following fixation, the cells were washed with $1 \%$ Triton X-100 in PBS (pH 7.4). Next, the cells were blocked in $1.5 \%$ horse serum in PBS for 30 min at room temperature, and then incubated overnight at $4^{\circ} \mathrm{C}$ with primary antibodies (1:50) for p-Mek, p-4EBP1, p-Erk (Cell Signaling Technology), p-Akt (Abcam, Cambridge, MA) and p-mTOR (eBioscience, San Diego, CA), in a humidified chamber. After washing twice with PBS, the cells were incubated with rabbit tetramethylrhodamine isothiocyanate (TRITC)-labeled secondary antibody (1:100, Dianova, Hamburg, Germany) for $1 \mathrm{~h}$ at room temperature. The cells were also stained with 4,6-diamidino-2-phenylindole (DAPI) to visualize the nuclei. The slides were then washed twice with PBS, and covered with Dabco (Sigma-Aldrich) before confocal laser scanning microscopy was performed (Olympus, Tokyo, Japan). 
Analysis of cytochrome c localization. AsPC-1 cells were plated on 18-mm glass coverslips with RPMI-1640 medium and grown to approximately $70 \%$ confluence for $24 \mathrm{~h}$. The cells were treated with gemcitabine $(1 \mu \mathrm{M})$ alone or in combination with HS-104 $(5 \mu \mathrm{M})$ for $6 \mathrm{~h}$, and then incubated with $500 \mathrm{nM}$ MitoTracker Red probe (Molecular Probes Inc., Eugene, OR) for $30 \mathrm{~min}$ at $37^{\circ} \mathrm{C}$. Next, the cells were washed twice with PBS, fixed in an acetic acid: $70 \%$ ethanol (1:2) solution for $10 \mathrm{~min}$ at $-20^{\circ} \mathrm{C}$, and then incubated overnight at $4^{\circ} \mathrm{C}$ with cytochrome $c$ antibody (1:50; Santa Cruz Biotechnologies, Santa Cruz, CA). On the following day, the cells were washed twice with PBS and incubated with mouse fluorescently labeled secondary antibody (1:100, Dianova) for $1 \mathrm{~h}$ at room temperature. The cells were also stained with DAPI to visualize the nuclei. The slides were then washed twice with PBS, and covered with Dabco (Sigma-Aldrich) before confocal laser scanning microscopy was performed (Olympus).

DAPI staining and TUNEL assay. AsPC-1 cells were plated on 18-mm glass coverslips with RPMI-1640 medium and grown to approximately $70 \%$ confluence for $24 \mathrm{~h}$. The cells were treated with gemcitabine $(1 \mu \mathrm{M})$ and/or HS-104 $(5 \mu \mathrm{M})$ for $24 \mathrm{~h}$, washed twice with PBS, and fixed in an acetic acid: $70 \%$ ethanol (1:2) solution for $10 \mathrm{~min}$ at $-20^{\circ} \mathrm{C}$. Following fixation, the cells were washed with $1 \%$ Triton X-100 in PBS (pH 7.4). Next, the cells were blocked in $1.5 \%$ horse serum in PBS for $30 \mathrm{~min}$ at room temperature, and then stained with $2 \mu \mathrm{g} / \mathrm{ml}$ of DAPI (Sigma-Aldrich) for $20 \mathrm{~min}$ at $37^{\circ} \mathrm{C}$. The stained cells were examined under a fluorescence microscope for evidence of nuclear fragmentation. TUNEL assay was performed using the In Situ Cell Death Detection Kit (Roche Co. Ltd, Mannheim, Germany).

Matrigel plug assay. All animal manipulations were performed in accordance with the animal care guidelines of the Guide for Animal Experiments by the Korean Academy of Medical Sciences. Male BALB/c 6-week-old mice were obtained from Orient-Bio Laboratory Animal Research Center Co., Ltd. (Kapyung, South Korea). The animals were fed with standard mice chow with free access to tap water in an animal house with controlled temperature $\left(21^{\circ} \mathrm{C}\right)$ and humidity and alternating $12 \mathrm{~h}$ light-dark cycles. Liquid Matrigel was mixed at $4^{\circ} \mathrm{C}$ with concentrated VEGF $(100 \mathrm{ng} / \mathrm{ml})$, and $1 \mu \mathrm{M}$ gemcitabine and/or $5 \mu \mathrm{M} \mathrm{HS}-104$ and/or PBS $(10 \mu \mathrm{l})$ were injected subcutaneously into the mice. Matrigel plugs were surgically harvested on day 7 and photographed. The plugs were fixed in $4 \%$ buffered formaldehyde, embedded in paraffin and sectioned. The 8- $\mu \mathrm{m}$-thick sections were stained with hematoxylin and eosin (H\&E). H\&E staining was performed to identify the formation and infiltration of new, functional microvessels. Functional vessels with intact RBCs were quantified manually by using a microscope [high-power field (HPF) x200 magnification].

Animal experiments. A total of $20 \mathrm{BALB} / \mathrm{c}$ nu/nu mice were intraperitoneally injected with a ketamine:Rumpun mixture (9:1, $25 \mu \mathrm{l} / \mathrm{mice})$ to induce anesthesia and were then arranged in the decubitus position. Then, $100 \mu \mathrm{l}$ of an AsPC-1 singlecell suspension $\left(1 \times 10^{6} \mathrm{ml}^{-1}\right)$ prepared using a 1-ml injector was surgically implanted into the pancreas, after which the needle was promptly pulled out. After injection, the mice were maintained in an animal house with controlled temperature $\left(21^{\circ} \mathrm{C}\right)$ and humidity and alternating $12 \mathrm{~h}$ light-dark cycles, and fed with standard mouse chow and had free access to tap water. One week after tumor implantation, the mice were randomly divided into 4 groups to receive gemcitabine and HS-104 formulations. One group of mice was left untreated, the second group received gemcitabine, the third group received HS-104, and the fourth group received both gemcitabine and HS-104. HS-104 was administered orally (20 mg/kg/day) as a 5\% w/v solution in DMSO:PEG 400:water (1:5:4, v/v), and gemcitabine was diluted in sterile saline and injected intraperitoneally ( $25 \mathrm{mg} / \mathrm{kg} /$ day) 5 times per week for 4 weeks. Body weight of the mice and tumor growth were monitored.

Immunohistochemistry. Immunostaining was performed using 8- $\mu$ m-thick sections of the tumor samples after deparaffinization. Microwave antigen retrieval was performed in citrate buffer ( $\mathrm{pH} \mathrm{6.0)}$ for $10 \mathrm{~min}$ prior to peroxidase quenching with $3 \%$ hydrogen peroxide $\left(\mathrm{H}_{2} \mathrm{O}_{2}\right)$ in PBS for $10 \mathrm{~min}$. The sections were then washed in water and preblocked with normal goat or horse serum for $10 \mathrm{~min}$. Next, the tissue sections were incubated overnight at $4^{\circ} \mathrm{C}$ in 1:50 dilutions of primary antibodies against PCNA, cleaved caspase-3, CD34 (Santa Cruz Biotechnology), p-Akt and p-Mek (Cell Signaling Technologies). The sections were then incubated with biotinylated secondary antibodies (1:100) for $1 \mathrm{~h}$. After washing with PBS, streptavidin-HRP was applied. Finally, the sections were developed with diaminobenzidine tetrahydrochloride substrate for $10 \mathrm{~min}$, and counterstained with hematoxylin. At least three random fields of each section were examined at a x400 magnification.

Pimonidazole staining. Hypoxic regions in mouse tissues were visualized using the Hydroxyprobe-1 kit (Chemicon International, Temecula, CA). These experiments were approved by the animal experimentation ethics committee, according to local and governmental regulations. Briefly, mice were injected intraperitoneally with pimonidazole $(60 \mathrm{mg} / \mathrm{kg}$ body weight), which forms adducts when reductively activated in hypoxic cells. Mice were sacrificed 90 min later by asphyxiation in $\mathrm{CO}_{2}$. The hydroxyprobe-1 monoclonal antibody was biotinylated using d-biotinyl- $\varepsilon$-aminocaproic acid $N$-hydroxysuccinimide ester (Boehringer, Mannheim, Germany) and was used to stain pimonidazole adducts in formalin-fixed paraffin-embedded tissues according to the manufacturer's instructions (25).

Statistical analysis. Data are expressed as the mean \pm SD, and were analyzed with an ANOVA and unpaired Student's t-test. A p-value $\leq 0.05$ was considered statistically significant. Statistical calculations were performed using SPSS software for the Windows operating system (version 10.0; SPSS, Chicago, IL).

\section{Results}

Gemcitabine and HS-104 synergistically inhibit the proliferation of pancreatic cancer cells. We evaluated the effect of the gemcitabine and HS-104 combination treatment on PANC-1 

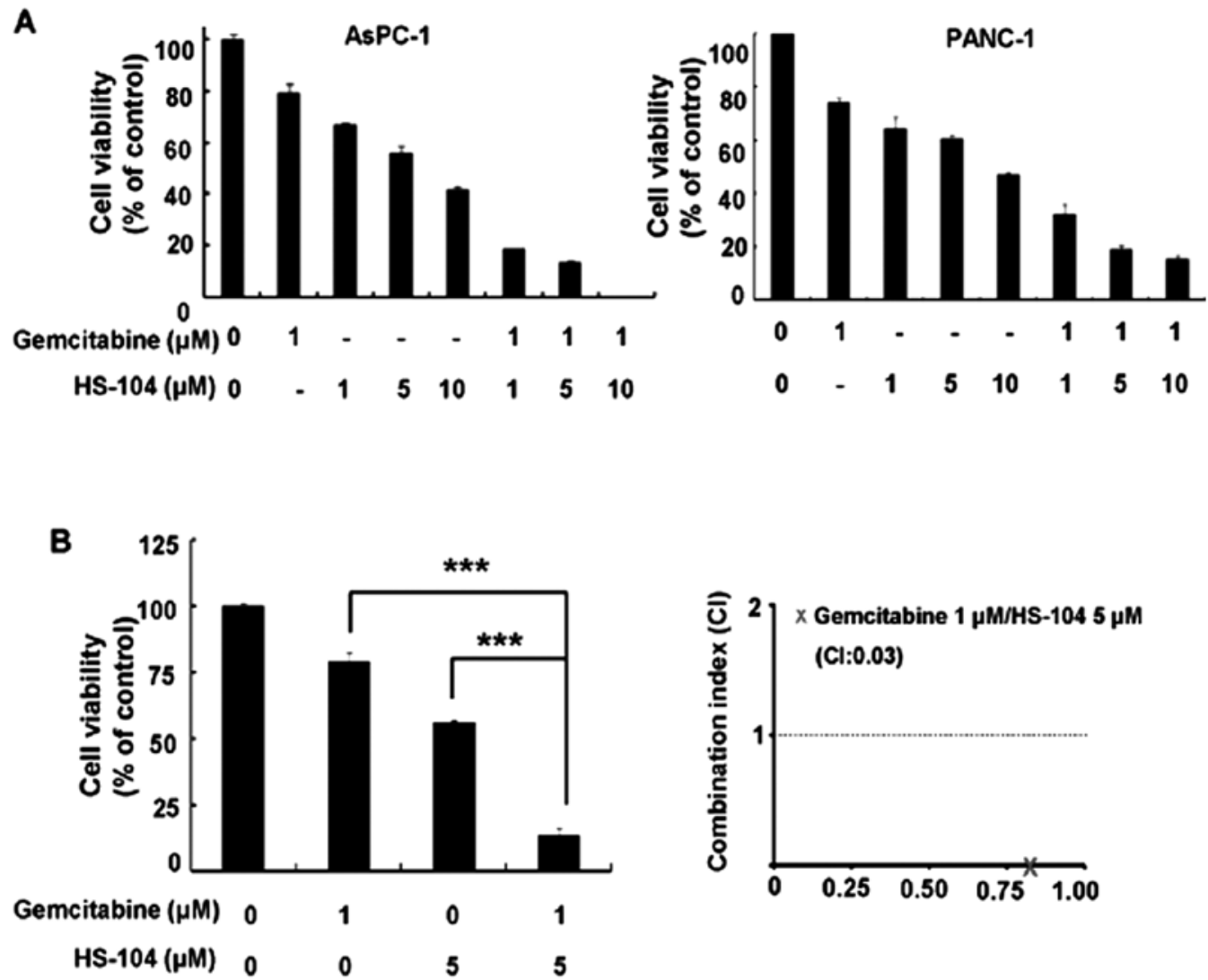

Figure 1. Synergistic cytotoxic effect of gemcitabine and HS-104 on AsPC-1 and PANC-1 pancreatic cancer cells. (A) AsPC-1 and PANC-1 cells were treated with gemcitabine $(1 \mu \mathrm{M})$ and/or HS-104 $(0,1,5$ or $10 \mu \mathrm{M})$ for $72 \mathrm{~h}$. MTT assay was performed to determine the cytotoxic effects of gemcitabine and HS-104. (B) Effect of combination treatment with $1 \mu \mathrm{M}$ gemcitabine and $5 \mu \mathrm{M} \mathrm{HS}-104$ on cell viability of AsPC-1 cells. The combination index (CI) values for gemcitabine $(1 \mu \mathrm{M})$ and HS-104 $(5 \mu \mathrm{M})$ in AsPC-1 cells were determined using the Chou-Talalay method and CalcuSyn software (Biosoft). A CI value of $<1$ was considered to be indicative of a synergistic effect. The combination of gemcitabine $(1 \mu \mathrm{M})$ and HS-104 $(5 \mu \mathrm{M})$ in AsPC-1 cells showed a synergistic effect. A CI value of 0.03 indicated synergism. Data are expressed as the mean \pm SD from triplicate wells. ${ }^{* * *} \mathrm{p}<0.001$.

and AsPC-1 pancreatic cancer cells by using a cell viability assay. The cells were treated with a fixed concentration of gemcitabine $(1 \mu \mathrm{M})$ alone or with various concentrations of HS-104 $(0,1,5$ or $10 \mu \mathrm{M})$ for $72 \mathrm{~h}$. Compared to treatment with either agent alone, the combination of gemcitabine and HS-104 significantly inhibited growth in the two human pancreatic cancer cell lines (Fig. 1A). To identify the synergistic effect between gemcitabine and HS-104, we further examined the combination index $(\mathrm{CI})$ values using CalcuSyn software. Indeed, a synergistic effect of gemcitabine and HS-104 was more significantly observed in the AsPC-1 cells (Fig. 1B). The $\mathrm{CI}$ values were $<1$ for the combination of $1 \mu \mathrm{M}$ gemcitabine and $5 \mu \mathrm{M}$ HS-104 in AsPC-1 cells ( $\mathrm{CI}=0.03)$.

Gemcitabine and HS-104 synergistically induce apoptosis. Since the combination treatment of gemcitabine and HS-104 caused a significant reduction in cell proliferation, we next investigated whether the treatment with gemcitabine and HS-104 induced apoptosis by performing DAPI and TUNEL staining in AsPC-1 cells. As shown in Fig. 2A, among the cells simultaneously treated with the two drugs, the number of TUNEL-positive cells was higher than that among the cells treated with each agent alone. We next performed cytochrome $c$ staining to identify the involvement of the combined treatment of gemcitabine and HS-104 in changes in mitochon- drial membrane potential (MMP), which induces the release of mitochondrial cytochrome $c$ into the cytosol, a hallmark of intrinsic pathway-mediated apoptosis. As shown in Fig. 2B, we observed that the combined treatment synergistically increased cytochrome $c$ release with a concomitant decrease in the colocalization of cytochrome $c$ and mitochondria. In addition, the combination treatment significantly increased the expression levels of cleaved caspase- 3 and Bax as well as decreased Bcl-2 expression in AsPC-1 cells (Fig. 2C). Collectively, these results indicate that the combination of gemcitabine and HS-104 synergistically induced apoptosis of pancreatic cancer cells.

The combination of gemcitabine and HS-104 inhibit key enzymes in both PISK/Akt and RAF/Mek signaling pathways. The PI3K/Akt pathway is highly activated in pancreatic cancer, consequently promoting cell proliferation, survival and tumorigenesis $(26,27)$. Additionally, the Ras kinase-mediated cascade is the most widely known pathway (28), and upregulation of this pathway has been thought to play an important role in pancreatic cancer cell growth (29). Hence, we examined the combination effect of HS-104 (a PI3K inhibitor) and gemcitabine (a clinically approved first-line anticancer drug) on the two given pathways in pancreatic cancer cells. HS-104 clearly inhibited the expression of p-Akt, p-mTOR and p-4EBP1, main mediators of the PI3K/Akt pathway, while gemcitabine weakly inhibited the 


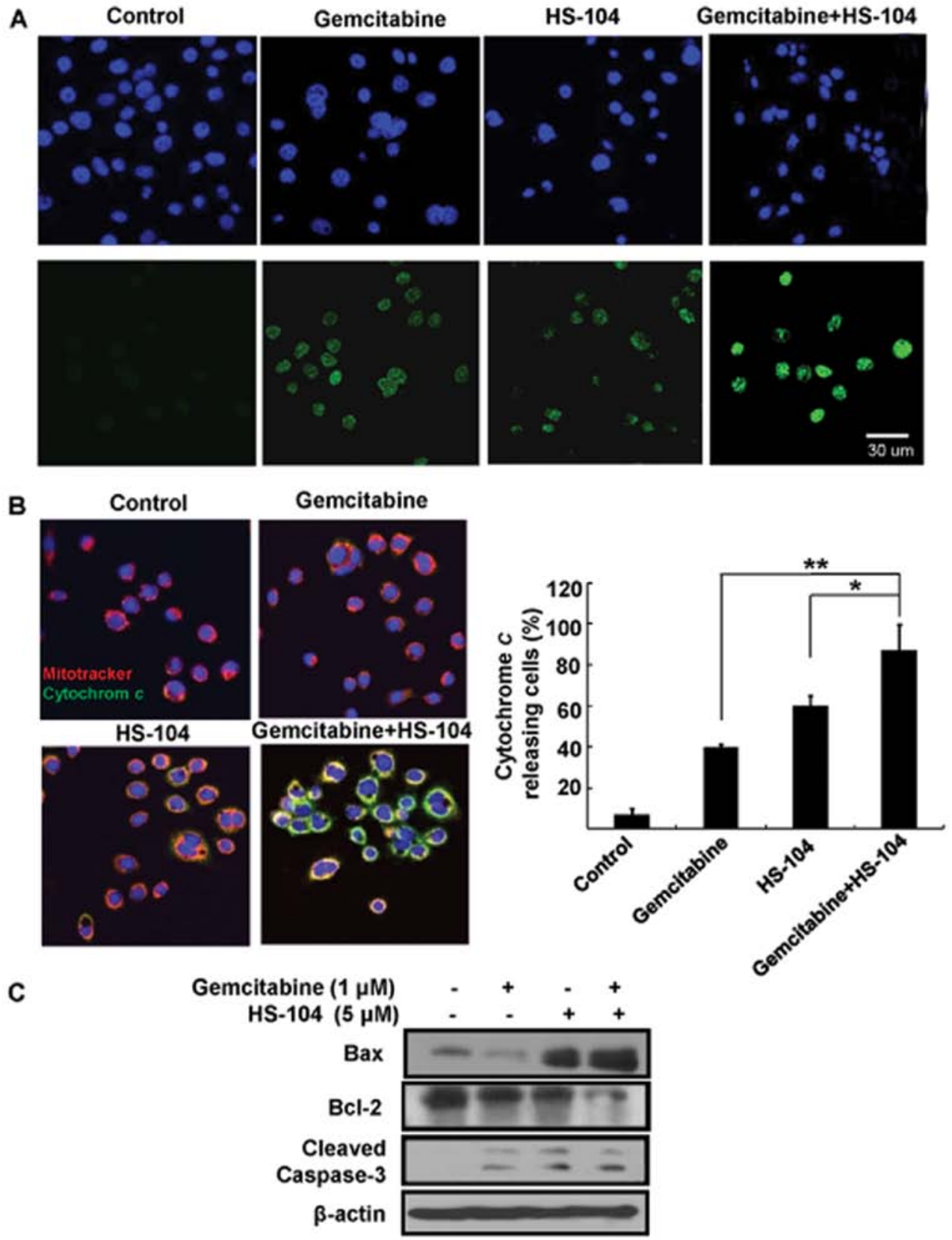

Figure 2. Combined treatment with gemcitabine and HS-104 induces synergistic apoptosis. (A) Induction of apoptosis by the combination of gemcitabine and HS-104 was determined in AsPC-1 cells by performing DAPI and TUNEL staining. (B) After treatment with $1 \mu \mathrm{M}$ gemcitabine and $5 \mu \mathrm{M} \mathrm{HS}-104$ for $24 \mathrm{~h}$, AsPC-1 cells were stained with anti-cytochrome $c$ antibody, MitoTracker and DAPI. The immunostained cells were analyzed under an Olympus confocal laser scanning microscope. For quantification of cells with diffuse cytochrome $c$ staining, we analyzed the percentage of cell that showed cytochrome $c$ release. (C) AsPC-1 cells were treated with gemcitabine $(1 \mu \mathrm{M})$ and/or HS-104 $(5 \mu \mathrm{M})$ for $72 \mathrm{~h}$. Cell lysates were prepared and subjected to western blot analysis for Bax, Bcl-2 and cleaved caspase-3. Data are expressed as the mean \pm SD from the three experiments. ${ }^{* *} \mathrm{p}<0.01$ vs. gemcitabine-treated group and ${ }^{*} \mathrm{p}<0.05$ vs. HS-104-treated group.

expressions of p-Akt, p-mTOR, p-Mek and p-Erk in AsPC-1 cells. However, compared to treatment with each agent alone, the combination treatment with both agents decreased the expression of p-Akt, p-mTOR, p-Mek and p-Erk, indicating synergistic suppression of both PI3K/Akt and RAF/Mek pathways (Fig. 3A). These results were confirmed using confocal fluorescent microscopy (Fig. 3B).

The combination of gemcitabine and HS-104 synergistically suppresses angiogenesis in an in vivo Matrigel plug assay. We further explored the anti-angiogenic activity of combined treatment of gemcitabine and HS-104 by using an in vivo Matrigel plug assay. Matrigel containing VEGF alone or with gemcitabine and/or HS-104 was subcutaneously injected into male BALB/c mice and removed 7 days after implantation. As shown in Fig. 4A, blood vessels were rarely observed in Matrigel plugs without VEGF. The Matrigel plugs containing VEGF alone appeared deep red in color because of the presence of red blood cells (RBCs), indicating that new blood vessels were formed inside the Matrigel via angiogenesis initiated by VEGF. However, compared to treatment with a single agent alone, the combination treatment of gemcitabine and 


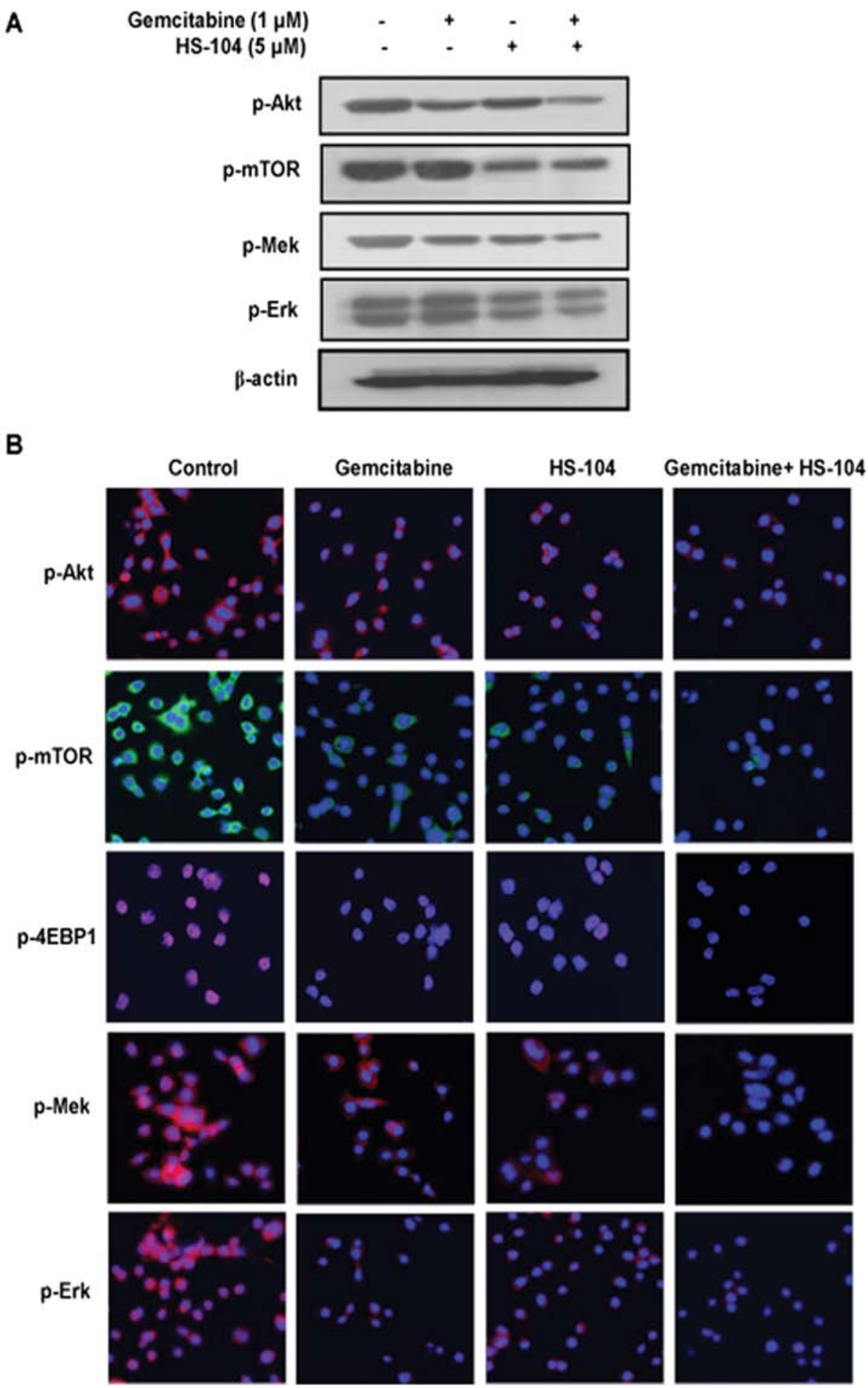

Figure 3. The combination of gemcitabine and HS-104 inhibits key enzymes in the PI3K/Akt and RAF/Mek signaling pathways. (A) Effects of gemcitabine and HS-104 on the expression levels of p-Akt, p-mTOR, p-Mek and p-Erk were determined by western blot analysis. AsPC-1 cells were treated with gemcitabine $(1 \mu \mathrm{M})$ and/or HS-104 $(5 \mu \mathrm{M})$ for $6 \mathrm{~h}$. (B) After treatment with $1 \mu \mathrm{M}$ gemcitabine and $5 \mu \mathrm{M}$ HS-104 for $6 \mathrm{~h}$ in AsPC-1 cells, the expression of p-Akt, p-mTOR, p-4EBP1, p-Mek and p-Erk was detected by immunofluorescence.

HS-104 considerably inhibited vascular formation (Fig. 4A). We performed H\&E staining to determine functional vasculature in the Matrigel plugs. As expected, the combination of gemcitabine and HS-104 suppressed VEGF-induced functional blood vessels formation to a greater degree than treatment with a single agent alone (Fig. 4B). These results suggest that the combination of gemcitabine with HS-104 showed a more potent inhibitory effect against VEGF-induced vessel formation in vivo than that shown by treatment with either agent alone.

The combination effects of gemcitabine with HS-104 in orthotopic pancreatic cancer animal models. To evaluate the antitumor efficacy of HS-104, alone and in combination with gemcitabine, we used orthotopic models of pancreatic cancer 
A

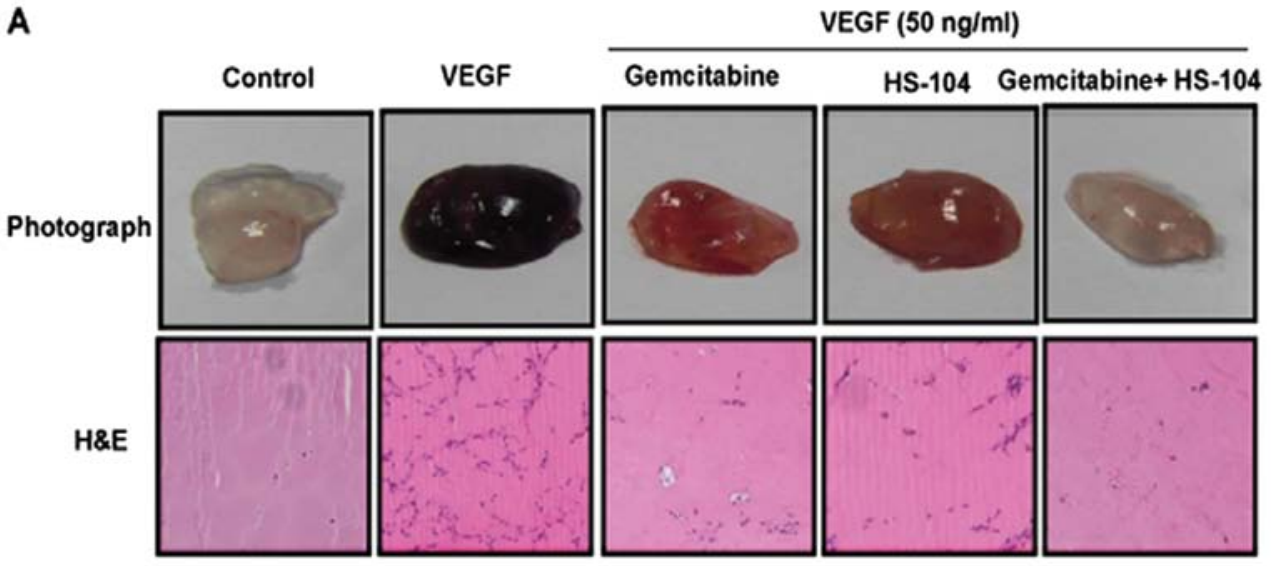

B

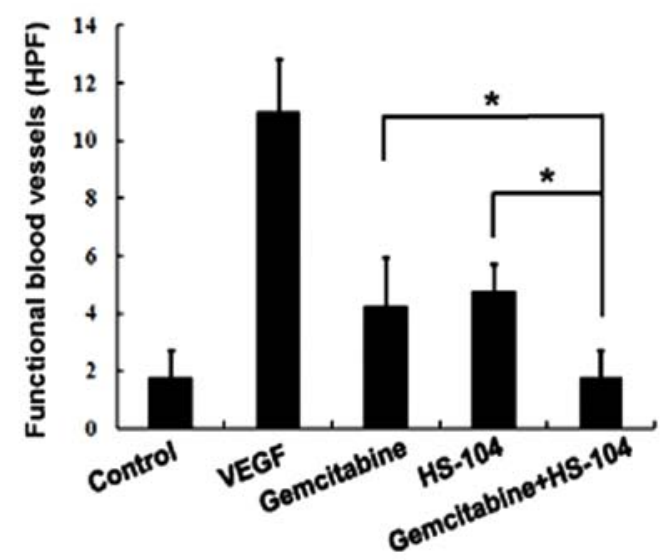

Figure 4. In vivo anti-angiogenic effects of the combination treatment with gemcitabine and HS-104 were evaluated using the Matrigel plug assay. (A) Matrigel plugs were implanted into mice with VEGF $(50 \mathrm{ng} / \mathrm{ml})$ and/or $1 \mu \mathrm{M}$ gemcitabine and/or $5 \mu \mathrm{M}$ HS-104. After 7 days, the plugs were removed, sectioned, stained with $\mathrm{H} \& \mathrm{E}$ and photographed to evaluate the extent of vascularization. (B) We used H\&E staining to determine functional vasculature in Matrigel plugs and found that the combination of two agents dramatically inhibited VEGF-induced vessel formation in vivo. " $\mathrm{p}<0.05$ vs. gemcitabine-treated group or HS-104-treated groups. Functional vessels were quantified manually using a microscope [high-power field (HPF) x200 magnification].

with AsPC-1 cells in BALB/c nu/nu mice. All treatments were well tolerated, with no significant differences in body weight among the treatment groups. The incidence of tumor formation was more than $95 \%$ after implantation in the pancreas. Treatment with HS-104 (20 mg/kg/day, p.o.) or gemcitabine $(25 \mathrm{mg} / \mathrm{kg} / \mathrm{day}$, i.p.) inhibited the growth of the primary pancreatic tumor by 33 or $23 \%$, respectively, compared with the control treatment. In particular, the antitumor effect was substantially enhanced when the two agents were combined, with $73 \%$ growth inhibition observed, compared to that observed with the control treatment (Fig. 5A).

The combination of gemcitabine with HS-104 inhibits proliferation and angiogenesis with induction of apoptosis in the orthotopic pancreatic cancer models. In a histopathological analysis, we observed that the combination treatment of HS-104 with gemcitabine synergistically decreased the expression of PCNA, a cell proliferation marker and CD34, an angiogenesis marker, compared with the control treatment. In addition, the combination treatment increased apoptotic cells in tumor tissues, as identified by expression of cleaved caspase- 3 and DNA fragments by performing the TUNEL assay (Fig. 5B). Owing to the antitumor effects of the combination treatment, we further examined the hypoxic regions in the tumors, which were visualized using a hypoxic probe (pimonidazole) that has been widely used to detect cellular and tissue hypoxia (30). Interestingly, the pimonidazole levels expectedly decreased because of the combination treatment compared to treatment with a single agent alone. Moreover, the combination treatment decreased the phosphorylation levels of Akt and Mek for cell survival and proliferation.

\section{Discussion}

Pancreatic cancer has one of the poorest prognoses among all cancers because of its tendency for late detection and its peculiar resistance to chemotherapy. Chemotherapeutic options for pancreatic cancer are limited, and the standard of care, gemcitabine, improves survival only minimally (31). Combination therapy is typically employed to achieve a better response rate than that achieved with monotherapy and is generally designed empirically by using drugs that act through different cytotoxic mechanisms with less overlapping toxicity. Clinical studies indicate that the combination of gemcitabine and various anticancer drugs is needed for survival prolongation, although previous uses of combination therapies have 
A

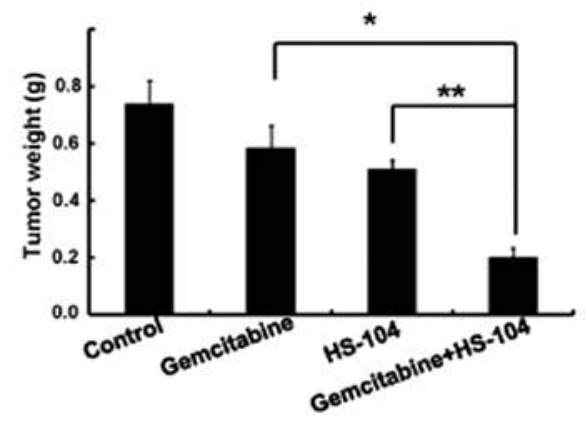

B

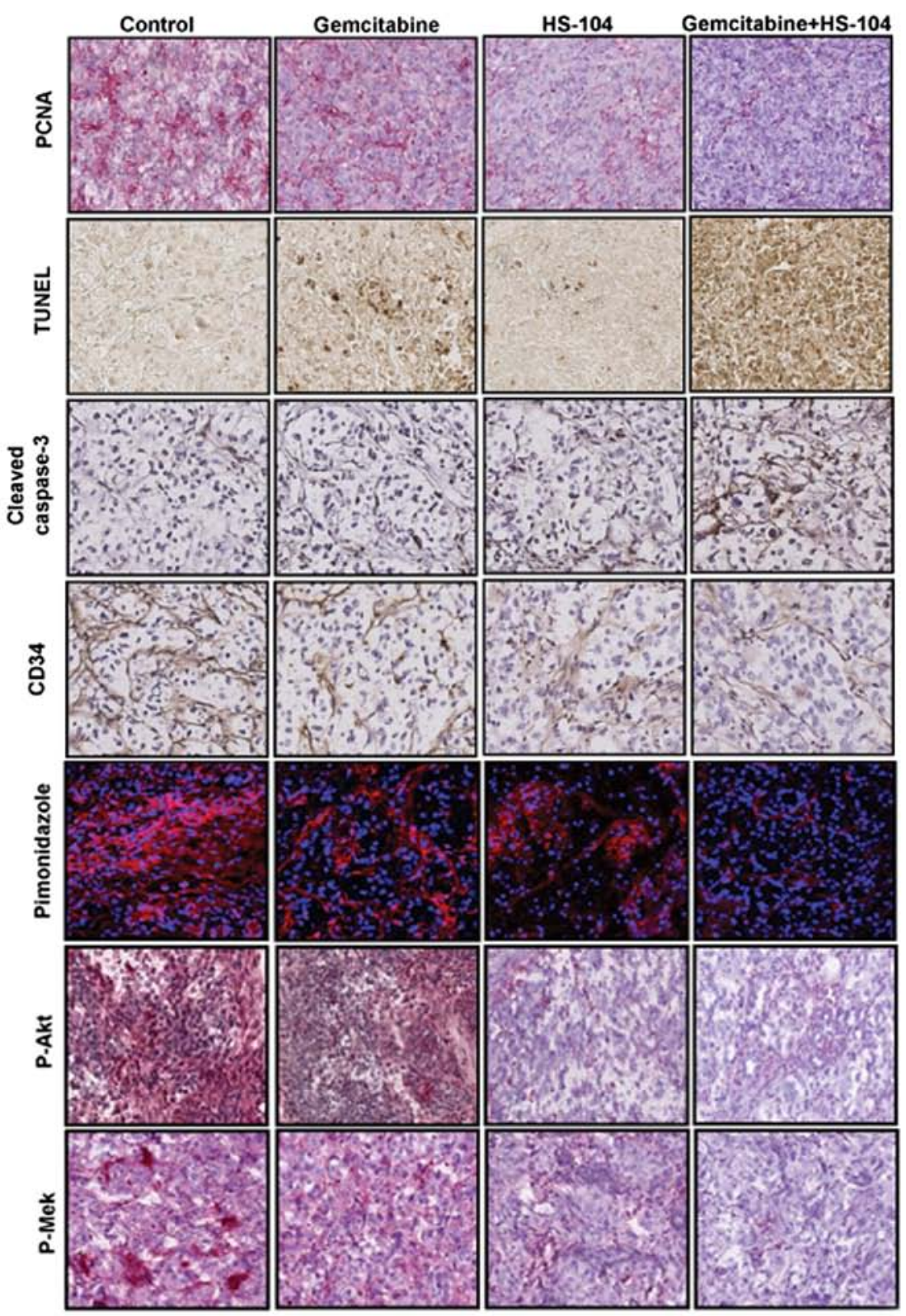

Figure 5. Effect of the combination treatment with gemcitabine and HS-104 in a pancreatic orthotopic mouse model. (A) Tumor weight of AsPC-1 pancreatic cancer orthotopic model established in nude mice. Pancreatic tumors were isolated from each treatment group after implantation of AsPC-1 pancreatic cancer cells. " $\mathrm{p}<0.05$ vs. gemcitabine-treated group and ${ }^{* *} \mathrm{p}<0.01$ vs HS-104-treated group. (B) In vivo effect of the combination of gemcitabine and HS-104 on proliferation, apoptosis and angiogenesis in a pancreatic orthotopic mouse model. Tumors were excised and processed for immunostaining for PCNA, TUNEL, cleaved caspase-3, CD34, pimonidazole, p-Akt and p-Mek including H\&E staining. x400 magnification.

not resulted in meaningful improvement. Currently, a major challenge is to identify new targets and more effective combi- nation therapies with gemcitabine, a first-line drug in the treatment of pancreatic cancer. 
Pancreatic cancers are associated with high incidence of K-ras mutations with increased signaling through the PI3K/Akt pathway and the cancer cells show high Akt activation (16), suggesting PI3K/Akt as a target for gemcitabine combinations. In addition, we previously reported that HS-104, a novel PI3K inhibitor, inhibited tumor growth by inhibiting PI3K/Akt signaling in hepatocellular carcinoma and breast cancer $(22,23)$. These findings have led us to investigate the combination effect of gemcitabine and HS-104 in pancreatic cancer. Our study showed that i) the combination of gemcitabine and HS-104 could decrease the concentrations of gemcitabine that were needed to inhibit pancreatic cancer cell growth and induce apoptosis; ii) apoptosis by the combination of gemcitabine and HS-104 was mediated by mitochondrial potential; iii) the combination of gemcitabine and HS-104 enhanced cell death by inhibiting PI3K/Akt signaling along with the Mek/Erk signaling pathway; and iv) the combination of gemcitabine and HS-104 synergistically inhibited tumor growth in orthotopic pancreatic cancer animal models.

The use of combinations of various agents, including gemcitabine, for treating pancreatic cancer has been reported previously. For instance, the combination of gemcitabine and 5-fluorouracil (5-FU) (32), as well as gemcitabine with cetuximab (33) or bevacizumab (34), failed to improve survival rates in pancreatic cancer patients. In this respect, our combination experiment of gemcitabine and HS-104 is a meaningful trial. Indeed, the present study showed that the combination of gemcitabine with HS-104 significantly inhibited the growth of pancreatic cancer cells compared to treatment with either agent alone. When we calculated CI values to further characterize the synergistic effect between gemcitabine and HS-104, the combination of $1 \mu \mathrm{M}$ gemcitabine with $5 \mu \mathrm{M}$ HS-104 was the most effective in AsPC-1 and PANC-1 cells. Since the PI3K/Akt signaling pathway is considered to be a viable and effective target for pancreatic cancer therapy $(35,36)$ and the inhibition of Akt can enhance the efficacy of gemcitabine chemotherapy in pancreatic cancer (37-39), we identified signaling pathways via which the combination of gemcitabine and HS-104 accelerated inhibition of cell growth in pancreatic cancer cells. We found that HS-104 inhibited substantially more the expression of p-Akt and p-mTOR than that of p-Mek and p-Erk, whereas gemcitabine weakly inhibited the expression of p-Akt, p-mTOR, p-Mek and p-Erk compared to inhibition by HS-104. However, the combination treatment decreased the expression of p-Mek, p-Erk, p-Akt, p-mTOR and p-4EBP1 more effectively than did treatment with either agent alone, indicating synergistic suppression of both the PI3K/Akt and RAF/Mek pathways. Considering these results, this synergistic effect by both agents is likely to be mediated by the ability of gemcitabine and HS-104 to inhibit both the PI3K/Akt and Raf/signaling pathways.

Apoptosis is induced by two alternative pathways: an extrinsic pathway mediated by the death receptor and the intrinsic pathway mediated by mitochondria. The extrinsic pathway involves cell surface death receptors, such as tumor necrosis factor or Fas, which upon binding of their ligands, initiate signaling to activated caspase- 8 , which cleaves caspase-3 directly to induce apoptosis. The intrinsic pathway is initiated by mitochondrial dysfunction and is regulated by members of the Bcl-2 family such as Bcl-2, Bax, Bak and Bid. In particular, a mitochondrial change activates the expression of members of the pro-apoptotic family and triggers the release of cytochrome $c$, which in turn activates caspase-9 and then caspase-3 in the cytosol (40). Our results showed that the combination of gemcitabine and HS-104 induced apoptosis of pancreatic cancer cells, which was observed as increased nuclear fragmentation upon TUNEL and DAPI staining. We identified the involvement of the combination treatment in mitochondrial membrane potential: the combination treatment induced marked changes in mitochondrial membrane potential and synergistically increased cytochrome $c$ release. In addition, it significantly increased the expression levels of Bax and cleaved caspase- 3 and decreased that of Bcl-2 in pancreatic cancer cells. The synergistic effect of the two agents on apoptosis was in line with the findings reported by Wei et al (41) and $\mathrm{Ng}$ et al (17), who showed that a combination of gemcitabine and PI3K inhibitors enhanced the induction of apoptosis in pancreatic cancer cells. Collectively, our data showed that the combination of gemcitabine with HS-104 synergistically induced mitochondria-mediated apoptosis in pancreatic cancer cells.

Most importantly, anticancer efficacy of the combination of gemcitabine and HS-104 in vitro was also observed in vivo in pancreatic cancer orthotopic animal models. Our study revealed that the combination of gemcitabine and HS-104 inhibited tumor growth without loss of body weight. These data are in accordance with induction of apoptosis due to increased expression of cleaved caspase-3 and DNA fragmentation, as observed in TUNEL staining, in parallel with a decrease in cell proliferation, as observed upon PCNA immunostaining in tumor tissues. Consequently, we considered that targeting PI3K/Akt signaling pathways with HS-104 may enhance the antitumor activity of gemcitabine, compared to treatment with either agent alone.

In conclusion, our results showed that a combination treatment of gemcitabine and HS-104 synergistically enhanced anticancer activity by inhibiting cell growth/proliferation and inducing apoptosis in vitro and in vivo, thereby indicating that induction of apoptosis and inhibition of cell proliferation by two agents may be a contributing factor for the suppression of tumor growth. In addition, our results showed that HS-104 may augment the therapeutic effect of gemcitabine in pancreatic cancer by direct or indirect inhibition of the PI3K/Akt signaling pathway, thereby leading to sensitization of pancreatic cancer cells to gemcitabine. Taken together, our findings suggest that the combination treatment with these two agents might be a candidate for future clinical application.

\section{Acknowledgements}

This research was supported by Inha University Grant, the Korean Health Technology R\&D Project (A120266, A110944), Ministry of Health and Welfare, and the National Research Foundation of Korea (NRF) funded by the Ministry of Education, Science and Technology (NRF-2012-0002988, 2012R1A2A2A01045602). 


\section{References}

1. Brothers HM II and Kostic NM: Catalytic activity of the serine proteases alpha-chymotrypsin and alpha-lytic protease tagged at the active site with a (terpyridine)platinum(II) chromophore. Biochemistry 29: 7468-7474, 1990.

2. Burris HA III, Moore MJ, Andersen J, Green MR, Rothenberg ML, Modiano MR, Cripps MC, Portenoy RK, Storniolo AM, Tarassoff P, Nelson R, Dorr FA, Stephens CD and Von Hoff DD: Improvements in survival and clinical benefit with gemcitabine as first-line therapy for patients with advanced pancreas cancer: a randomized trial. J Clin Oncol 15: 2403-2413, 1997.

3. Moore MJ, Goldstein D, Hamm J, Figer A, Hecht JR, Gallinger S, Au HJ, Murawa P, Walde D, Wolff RA, Campos D, Lim R, Ding K, Clark G, Voskoglou-Nomikos T, Ptasynski M and Parulekar W: Erlotinib plus gemcitabine compared with gemcitabine alone in patients with advanced pancreatic cancer: a phase III trial of the National Cancer Institute of Canada Clinical Trials Group. J Clin Oncol 25: 1960-1966, 2007.

4. Van Cutsem E, Vervenne WL, Bennouna J, Humblet Y, Gill S, Van Laethem JL, Verslype C, Scheithauer W, Shang A, Cosaert $\mathbf{J}$ and Moore MJ: Phase III trial of bevacizumab in combination with gemcitabine and erlotinib in patients with metastatic pancreatic cancer. J Clin Oncol 27: 2231-2237, 2009.

5. McGinn CJ, Zalupski MM, Shureiqi I, Robertson JM, Eckhauser FE, Smith DC, Brown D, Hejna G, Strawderman M, Normolle D and Lawrence TS: Phase I trial of radiation dose escalation with concurrent weekly full-dose gemcitabine in patients with advanced pancreatic cancer. J Clin Oncol 19: 4202-4208, 2001.

6. Hidalgo M: Pancreatic cancer. N Engl J Med 362: 1605-1617, 2010.

7. Vivanco I and Sawyers CL: The phosphatidylinositol 3-kinase AKT pathway in human cancer. Nat Rev Cancer 2: 489-501, 2002.

8. Cheng JQ, Lindsley CW, Cheng GZ, Yang H and Nicosia SV: The Akt/PKB pathway: molecular target for cancer drug discovery. Oncogene 24: 7482-7492, 2005.

9. Martelli AM, Faenza I, Billi AM, Manzoli L, Evangelisti C, Fala F and Cocco L: Intranuclear 3'-phosphoinositide metabolism and Akt signaling: new mechanisms for tumorigenesis and protection against apoptosis? Cell Signal 18: 1101-1107, 2006.

10. Agbunag C and Bar-Sagi D: Oncogenic K-ras drives cell cycle progression and phenotypic conversion of primary pancreatic duct epithelial cells. Cancer Res 64: 5659-5663, 2004.

11. Korc M: Role of growth factors in pancreatic cancer. Surg Oncol Clin N Am 7: 25-41, 1998.

12. Vincent F, de Boer J, Pfohl-Leszkowicz A, Cherrel Y and Galgani F: Two cases of ras mutation associated with liver hyperplasia in dragonets (Callionymus lyra) exposed to polychlorinated biphenyls and polycyclic aromatic hydrocarbons. Mol Carcinog 21: 121-127, 1998.

13. Bardeesy $\mathbf{N}$ and DePinho RA: Pancreatic cancer biology and genetics. Nat Rev Cancer 2: 897-909, 2002.

14. Tong Z, Fan Y, Zhang W, Xu J, Cheng J, Ding M and Deng H: Pancreas-specific Pten deficiency causes partial resistance to diabetes and elevated hepatic AKT signaling. Cell Res 19: 710-719, 2009

15. Ali S, Banerjee S, Ahmad A, El-Rayes BF, Philip PA and Sarkar FH: Apoptosis-inducing effect of erlotinib is potentiated by 3,3'-diindolylmethane in vitro and in vivo using an orthotopic model of pancreatic cancer. Mol Cancer Ther 7: 1708-1719, 2008.

16. Izuishi K, Kato K, Ogura T, Kinoshita T and Esumi H: Remarkable tolerance of tumor cells to nutrient deprivation: possible new biochemical target for cancer therapy. Cancer Res 60: 6201-6207, 2000.

17. Ng SSW, Tsao MS, Chow S and Hedley DW: Inhibition of phosphatidylinositide 3-kinase enhances gemcitabine-induced apoptosis in human pancreatic cancer cells. Cancer Res 60: 5451-5455, 2000.

18. Yokoi $\mathrm{K}$ and Fidler IJ: Hypoxia increases resistance of human pancreatic cancer cells to apoptosis induced by gemcitabine. Clin Cancer Res 10: 2299-2306, 2004.

19. Bondar VM, Sweeney-Gotsch B, Andreeff M, Mills GB and McConkey DJ: Inhibition of the phosphatidylinositol 3'-kinaseAKT pathway induces apoptosis in pancreatic carcinoma cells in vitro and in vivo. Mol Cancer Ther 1: 989-997, 2002.
20. Venkannagari S, Fiskus W, Peth K, Atadja P, Hidalgo M, Maitra A and Bhalla KN: Superior efficacy of co-treatment with dual PI3K/mTOR inhibitor NVP-BEZ235 and pan-histone deacetylase inhibitor against human pancreatic cancer. Oncotarget 3: 1416-1427, 2012 .

21. Kim O, Jeong Y, Lee H, Hong SS and Hong S: Design and synthesis of imidazopyridine analogues as inhibitors of phosphoinositide 3-kinase signaling and angiogenesis. J Med Chem 54: 2455-2466, 2011

22. Jung KH, Zheng HM, Jeong Y, Choi MJ, Lee H, Hong SW, Lee HS, Son MK, Lee S, Hong S and Hong SS: Suppression of tumor proliferation and angiogenesis of hepatocellular carcinoma by HS-104, a novel phosphoinositide 3-kinase inhibitor. Cancer Lett 328: 176-187, 2013.

23. Lee H, Li GY, Jeong Y, Jung KH, Lee JH, Ham K, Hong S and Hong SS: A novel imidazopyridine analogue as a phosphatidylinositol 3-kinase inhibitor against human breast cancer. Cancer Lett 318: 68-75, 2012.

24. Lee KH, Lee JH, Han SW, Im SA, Kim TY, Oh DY and Bang YJ: Antitumor activity of NVP-AUY922, a novel heat shock protein 90 inhibitor, in human gastric cancer cells is mediated through proteasomal degradation of client proteins. Cancer Sci 102: 1388-1395, 2011

25. Greijer AE, Delis-van Diemen PM, Fijneman RJ, Giles RH, Voest EE, van Hinsbergh VW and Meijer GA: Presence of HIF-1 and related genes in normal mucosa, adenomas and carcinomas of the colorectum. Virchows Arch 452: 535-544, 2008.

26. Campbell PM, Groehler AL, Lee KM, Ouellette MM, Khazak V and Der CJ: K-Ras promotes growth transformation and invasion of immortalized human pancreatic cells by Raf and phosphatidylinositol 3-kinase signaling. Cancer Res 67: 2098-2106, 2007.

27. Ruggeri BA, Huang L, Wood M, Cheng JQ and Testa JR: Amplification and overexpression of the AKT2 oncogene in a subset of human pancreatic ductal adenocarcinomas. Mol Carcinog 21: 81-86, 1998.

28. Sridhar SS, Hedley D and Siu LL: Raf kinase as a target for anticancer therapeutics. Mol Cancer Ther 4: 677-685, 2005.

29. Siu LL, Awada A, Takimoto CH, Piccart M, Schwartz B, Giannaris T, Lathia C, Petrenciuc O and Moore MJ: Phase I trial of sorafenib and gemcitabine in advanced solid tumors with an expanded cohort in advanced pancreatic cancer. Clin Cancer Res 12: 144-151, 2006.

30. Doege K, Heine S, Jensen I, Jelkmann W and Metzen E: Inhibition of mitochondrial respiration elevates oxygen concentration but leaves regulation of hypoxia-inducible factor (HIF) intact. Blood 106: 2311-2317, 2005.

31. Fujioka S, Niu J, Schmidt C, Sclabas GM, Peng B, Uwagawa T, Li Z, Evans DB, Abbruzzese JL and Chiao PJ: NF-kappaB and AP-1 connection: mechanism of NF-kappaB-dependent regulation of AP-1 activity. Mol Cell Biol 24: 7806-7819, 2004.

32. Reni M, Balzano G, Aprile G, Cereda S, Passoni P, Zerbi A, Tronconi MC, Milandri C, Saletti P, Rognone A, Fugazza C, Magli A, Di Muzio N, Di Carlo V and Villa E: Adjuvant PEFG (cisplatin, epirubicin, 5-fluorouracil, gemcitabine) or gemcitabine followed by chemoradiation in pancreatic cancer: a randomized phase II trial. Ann Surg Oncol 19: 2256-2263, 2012.

33. Philip PA, Benedetti J, Corless CL, Wong R, O'Reilly EM, Flynn PJ, Rowland KM, Atkins JN, Mirtsching BC, Rivkin SE, Khorana AA, Goldman B, Fenoglio-Preiser CM, Abbruzzese JL and Blanke CD: Phase III study comparing gemcitabine plus cetuximab versus gemcitabine in patients with advanced pancreatic adenocarcinoma: Southwest Oncology Groupdirected intergroup trial S0205. J Clin Oncol 28: 3605-3610, 2010.

34. Kindler HL, Niedzwiecki D, Hollis D, Sutherland S, Schrag D, Hurwitz H, Innocenti F, Mulcahy MF, O'Reilly E, Wozniak TF, Picus J, Bhargava P, Mayer RJ, Schilsky RL and Goldberg RM: Gemcitabine plus bevacizumab compared with gemcitabine plus placebo in patients with advanced pancreatic cancer: phase III trial of the Cancer and Leukemia Group B (CALGB 80303). J Clin Oncol 28: 3617-3622, 2010.

35. Yotsumoto F, Fukami T, Yagi H, Funakoshi A, Yoshizato T, Kuroki M and Miyamoto S: Amphiregulin regulates the activation of ERK and Akt through epidermal growth factor receptor and HER3 signals involved in the progression of pancreatic cancer. Cancer Sci 101: 2351-2360, 2010. 
36. He L, Wu Y, Lin L, Wang J, Chen Y, Yi Z, Liu M and Pang X: Hispidulin, a small flavonoid molecule, suppresses the angiogenesis and growth of human pancreatic cancer by targeting vascular endothelial growth factor receptor 2-mediated $\mathrm{PI} 3 \mathrm{~K} / \mathrm{Akt} / \mathrm{mTOR}$ signaling pathway. Cancer Sci 102: 219-225, 2011.

37. Liu D, Zhang Y, Dang C, Ma Q, Lee W and Chen W: siRNA directed against TrkA sensitizes human pancreatic cancer cells to apoptosis induced by gemcitabine through an inactivation of PI3K/Akt-dependent pathway. Oncol Rep 18: 673-677, 2007.

38. Yao J and Qian C: Inhibition of Notch3 enhances sensitivity to gemcitabine in pancreatic cancer through an inactivation of PI3K/ Akt-dependent pathway. Med Oncol 27: 1017-1022, 2010.
39. Zhang B, Shi ZL, Liu B, Yan XB, Feng J and Tao HM: Enhanced anticancer effect of gemcitabine by genistein in osteosarcoma: the role of Akt and nuclear factor-kappaB. Anticancer Drugs 21: 288-296, 2010.

40. Soengas MS, Capodieci P, Polsky D, Mora J, Esteller M, Opitz-Araya X, McCombie R, Herman JG, Gerald WL, Lazebnik YA, Cordon-Cardo C and Lowe SW: Inactivation of the apoptosis effector Apaf-1 in malignant melanoma. Nature 409: 207-211, 2001.

41. Wei WT, Chen H, Wang ZH, Ni ZL, Liu HB, Tong HF, Guo HC, Liu DL and Lin SZ: Enhanced antitumor efficacy of gemcitabine by evodiamine on pancreatic cancer via regulating PI3K/Akt pathway. Int J Biol Sci 8: 1-14, 2012. 